\title{
Performance of Fractal Image Compression for Medical Images: A Comprehensive Literature Review
}

\author{
Amit Kumar Biswas \\ (PhD Research Scholar) \\ Department of \\ Computer Applications \\ Bhilai Institute of \\ Technology, Durg
}

(C.G.), India

\author{
Sanjeev Karmakar \\ Department of \\ Computer Applications \\ Bhilai Institute of \\ Technology, Durg \\ (C.G.), India
}

\author{
Sanjay Sharma \\ Department of Engg \\ Mathematics \\ Bhilai Institute of \\ Technology, Durg \\ (C.G.), India
}

\author{
Manoj Kumar \\ Kowar \\ Department of \\ Electronics \& \\ Telecommunications \\ Bhilai Institute of \\ Technology, Durg \\ (C.G.), India
}

\begin{abstract}
Generally the fractal image compression is a technique based on the presentation of an image by a contractive transform, on the space of image, for which the fixed point is close to the original image. The fractal image compression is rapidly growing principle covers a wide variety of coding scheme in various domains. A large number of theoretical aspects of this concept are available. However, a few concentrations have been given to the image encoding model which justifies its proper implementation. Most fractal base schemes are not competitive with the current state of the art like JPEG, JPEG2000. To identify the performance of fractal image compression, specifically for the medical images, a comprehensive review of worldwide contributions from 1990 to 2013 has been carried out. As a result, the fractal image compression techniques are classified into four domains, i.e., spatial, frequency, soft computing, and hybrid. It is found that, fractal theory in spatial domain, fractal theory along with discrete cosine transform and wavelet transform in frequency domain, fractal theory along with fuzzy logic and neural network in soft computing domain, and combination of fractal theory along with discrete cosine transform, wavelet transform, fuzzy logic and neural network in hybrid domain are often used by the contributors. It is found that the fractal dimension and fuzzy logic approach on regular image processing are sufficiently suitable for image texture analysis. Consequently fuzzy logic is successfully applied in regular image and found outstanding results. On the basis of these facts it is anticipated that the fractal dimension and fuzzy logic could be a suitable approach in medical image compression using fractal theory as a future expansion is discussed in this review article.
\end{abstract}

\section{Keywords}

Fractal image compression; fractal dimension; fuzzy logic; discrete cosine transform

\section{INTRODUCTION}

The fundamental principle of fractal image compression (FIC) is to represent an image by contractive transform in the coded form, where the fixed point is close to that image. Fixed point theory given by Banach is explain that, within a complete metric space, the fixed point of such a transform may be recovered by apply the iterative process to an arbitrary initial element of that space [1]. According to [2] [3], metric space images are representing by vectors in a Hilbert space. Image encoding is not a simple process, because there is no known algorithm for constructing the transform with the smallest possible distance between the corresponding fixed point and the image to be encoded. The common approach is based on the collage theorem which provides the distance between the image to be encoded and the fixed point of a transform, in terms of the minimum error distance between the transform of the image and the image itself. Therefore a suitable transform of a whole image may be reconstructed as a "collage" or union of mappings from the images to itself with a small "collage error" (the distance between the collage and the image).

According to [3][4], the above transform was generated by the integration of affine mappings on the entire image known as Iterated Function System (IFS). In, [4] [5] various image modeling were established by using IFS method and no one were developed any automated encoding algorithm. Fractal image compression (FIC) was practically implemented with the introduction of Partitioned IFS (PIFS) by Jacquin [3]. The PIFS differs from IFS by each of the individual mappings operates on a partition of the image, rather than the whole image. Since the image is partition into range blocks and domain block, where, each range block is mapped from one of the domain blocks as depicted in Figure 1, the integration of mappings constitute a transform on the image as a whole. The transform with minimum collage error is constructed by individual minimum collage error for each range block mapping. This transform is them represented by specifying the positions of the matching domain block together with the block mapping parameters as scaling (contrast) and offset (luminance). The distance are usually measured by the mean squared error (MSE), equivalent to the distance derived from the $l^{2}$ inner product given by [1], since optimization of the standard block mapping is simple under this measure by [3].

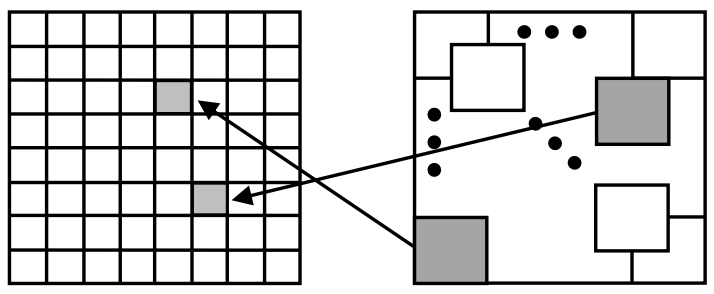

(a)

(b)

Fig. 1: One of the block mapping in a PIFS representation: (a) Range Blocks (b) Domain Blocks 
The fundamental principle of FIC clearly provides extensive scope in the design of a medical images compression by the implementation of FIC. Within this broad research scopes, the differences between the majorities of existing FIC schemes may classified into the following domains:

1. Spatial Domain

2. Frequency Domain

3. Fuzzy Domain

4. Hybrid Domain

For all the above domains, the following major aspects are considered in the fractal coding schemes:

1. The partition criteria for the range blocks

2. The preparation of the domain pool.

3. The classification approaches of domain blocks to reduce the search time.

4. The efficient searching method to locating suitable domain blocks.

5. The representation and quantization of the transform parameters

There are many theoretical results have been proposed for the design of the above aspect. In addition, the above aspects are not independent, in the sense that any comparative analysis of coding performance between different options in one of the above aspects is usually dependent on the corresponding choice in the other aspects. Consequently, a significant comparison between the relative merits of particular choice in each aspect is very difficult. This review paper is mainly intended as an overview of the variety of schemes that have been investigated in the four different domains and find the suitable domain(s) for the medical image compression using fractal theory.

In this review paper, coding of grayscale images has been considered. In [3], a collection of significant work by a number of authors have been explained, while [6] combines introductory material with an in-depth discussion of many aspects of fractal coding.

The objective of this comprehensive study is to identify the performance of FIC for medical images as well as specification and expansion of the future works. This objective is considered through a comprehensive review of worldwide contributions from 1990 to 2013. The review discussed mainly concerning the different approaches to reduce the encoding time in various domains. The paper is constructed with the various sections. Wherein, section II is discussed the review of literature in brief. Results and future expansion using fractal dimension (FD) and fuzzy logic (FL) is discussed in the section III. And finally conclusions of the study are discussed in the section IV.

\section{LITERATURE REVIEW}

Fractal theory was practically implemented by [4] on images using iterative contractive process given in [7][8].

A review paper [9] was published to describe an approach of image coding based on a fractal theory of iterated contractive transformations defined piecewise.
The general encoding and decoding system is based on the construction, for an original image to encode, of a fractal code- a contractive image transformation for which the original image is an approximate fixed point and when it applied iteratively on any initial image at the decoder, produces a sequence of images which converges to a fractal approximation of the original image. The fractal code consists of a description of both an image partition and a contractive image transformation defined as position of domain block mapping along with a small set of quantized parameters.

For the best encoding time and high reconstructed image quality, various encoding and decoding techniques have been developed for different domains. The following subsections briefly describe wide literature survey in the different domain area. The spatial domain describes the statistical, entropy, vector quantization, best searching, FD (block complexity measure) and other approaches. The frequency domain mainly describes the discrete cosine transform (DCT) and wavelet approaches. Soft-computing domain describes the fuzzy approach including image complexity, clustering, FD, neural network and genetic algorithm approaches for block classification, while the hybrid domain describes the incorporation of spatial domain, frequency domain and soft-computing domain approaches.

\subsection{Spatial Domain}

An image is representing in 2D by the functions $f(x, y)$ in spatial coordinates $(x, y)$ in an image plane. In spatial domain, the pixel composing of image details are considered and the various procedures are directly applied on these pixels. The image processing functions in the spatial domain may be expressed as

$$
g(x, y)=T\{f(x, y)\}
$$

where, $f(x, y)$ is the input image, $g(x, y)$ is the processed output image and $T$ represents an operation on ' $f$ ' defined over some neighborhood of $(x, y)$. Sometimes, $T$ can also be used to operate on a set of input images.

In statistical approach, the fractal image coding by block based classification method using statistics to accelerate the encoding time were proposed by [10] [11] [12] Similarly, [13] [14] were used the other statistic parameters called variance and mean on blocks to classify them for fractal coding, [15] was applied the mean of range and domain blocks to reduce the decoding time and [16] was applied the spatial correlation on range and domain blocks to decrease the encoding time. [17] [18] were proposed block based merging technique for image encoding with good quality reconstructed image.

Applied the vector quantization techniques with IFS to accelerate the search for the best domain block by [19][20] and also applied this method along with variance to search only the maximal intra-block variance quadrants by [21] and FD approach to partition the image using Local FD was proposed by [22]. In vector approach, proposed a suitable domain search technique based on feature extraction by [23] and applied the delaunay triangulation approach for split and merge technique along with vector quantization to speed-up the encoding process by [24].

Because of the IFS, cannot change the entropy values of image block, the entropy approach were applied on fractal 
coding to improve the compression time by [25][26][27] [28]. In gray-level difference based range-domain matching to perform the coding only when the range block gray level difference is less than the domain block gray level difference were given by [25][26].

The searching criterion is the important factor of FIC to speed up the encoding process. To improve the encoding time, proposed a multidimensional nearest neighbor search to search the suitable domain block using fixed partition by [27][28][29][30], using geometric partition by [31], using quadtree partition by [32][33] and region based heuristic search was proposed by [34]. Similarly, used the horizontal-vertical and variable sized split and merge partition method by [35][36].

For the acceleration of fractal image encoding time, used a maximum gradient technique by [37], used a fast no search algorithm based on a modified gray level transform using quadtree partition approach by [38]. Similarly, fractal coding using single kick-out condition and the zero contrast prediction was given by [39]. Normalized onenorm by [40], and normalize with kick-out condition was proposed by [41].

An evolutionary computing approach was proposed by [42] and a dynamic fractal transform approach proposed by [43] as well as a fractal theory for image compression was proposed by [44]. Similarly, the particle swarm optimization method to speed-up the encoding process was proposed by [45][46] and the Hubber M-estimation method for similarity measure of range and domain block with particle swarm optimization was utilized by [47].

Various methods have been proposed to measure the block complexity. FD is one the method to identify the less texture and more texture blocks. Geometric primitives that are self-similar and irregular in nature are termed as fractals. Fractal Geometry was introduced by Mandelbrot and has used over the years due to its broad spectrum of applications domains in [48]. In most of the applications, the common interest is to determine the FD of an object under investigation. In this direction, the FD to analysis the texture of the digital image and segmented it for coding using fractal theory was used by [17]

In other ways, a simple binary approach of square and rectangular block partition to encode the image was proposed by [49] and also proposed a modified FIC for binary images by [50]. An adaptive search process was applied to locate the best domain block by [51] and proposed an analysis paper for hybrid fractal image coding schemes by [52]. The parallel processing for FIC was implemented by [53] and the futility of square isometric on FIC was tested by [54]. With the visual properties of images, were used the visual property like edge in FIC were used by [55][56]. The Hilbert space-filling curves approach with run length encoding, LZ77 Coding, LZW coding and Huffman coding in medical image compression was used by [57].

\subsection{Frequency Domain}

The frequency domain is a space in which each image value at image position $F$ represents the amount that the intensity values in image $I$ vary over a specific distance related to $F$. In the frequency domain, changes in image position correspond to changes in the spatial frequency, (or the rate at which image intensity values) are changing in the spatial domain image $I$.

The frequency domain refers to the plane of the two dimensional discrete Fourier transform of an image. The purpose of the Fourier transform is to represent a signal as a linear combination of sinusoidal signals of various frequencies. In frequency domain, DCT is one of the frequently used techniques for image compression like JPEG. Thus, the characteristics of DCT approach were applied for domain block matching to accelerate the encoding process using fractal theory by [58]. Range and domain block matching based on texture or shape of the blocks by [59] and based on inner product by [60]. The law of cosine for the distance and angle between range and domain block matching were used by [61][62] and used the DCT coefficients to classify the domain blocks by [63] [64]. Similarly, applied the statistical approach on DCT coefficients of image block for high-quality image compression was proposed by [65]. In the similar context, used the fast Fourier transform based convolution approach by [66] and proposed a modified FIC using discrete fractal transform by [67]. A wavelet based image compression was proposed by [68] to encode the image without search; a wavelet based analysis of FIC by [69] and classified the domain blocks in wavelet domain by [70]. Used the wavelet to code the low frequency components and partition the high frequency energy of image by [71] and proposed a comparative paper of pure fractal and wavelet based FIC by [72]. A new wavelet based fractal image coding based on the initial error occur at different levels of sublevel based wavelet coefficients and the size of the sublevel and super blocks were changed according to the initial error by [73]. A simple classification approach in frequency domain to reduce the two groups of computation into one and reduce the encoding time was proposed by [74].

\subsection{Soft-Computing Domain}

The concepts related to image analysis contain a certain amount of uncertainty. It is well known for example that the definition of border pixels is a hard task. The decision whether a pixel belongs to the background or to the object is non-trivial on occasion due to the uncertainty present on object edges. For the robust encoding system, developments of new algorithms that involve softcomputing including fuzzy logic, neural network and genetic algorithm are required. This is why incorporating of soft-computing approach into the development of image processing and analysis for fractal encoding has been proposed in various research papers.

Implementation of fuzzy approach in image compression, a new method was proposed to describe the natural texture of image using fuzzy based FD and classified them by [75]. Cluster the similar image blocks into different groups using context sensitive fuzzy clustering approach by [76]. The image complexity also measured by the edge levels of the image using fuzzy approach were given by [77] [78] The applications of fuzzy logic techniques for image binarization, edge detection and geometric measurement were given by [79]. The fuzzy inference system based contrast enhancement using partial histogram by [80] and used the fuzzy sets to enhance the image by [81]. The fuzzy classifier and genetic algorithm based faster algorithm for FIC was proposed by [82]. 
After applying neural network approach in image processing, the neural network based domain classification methods for FIC were proposed by [83][84][85][86][87].

In the similar context, the genetic algorithm for fractal compression of images and videos with variable encoding time by changing parameters like population size and number of generation was proposed by [88]. Similarly, FIC based on classification using genetic algorithm by [89] and used the schema theorem to adapt the genetic operation by [90]. A study based research paper of FIC using genetic algorithm was given in [91]. A neural network approach was proposed to classify the partial discharge pattern by [92] and the fuzzy approach along with neural network by [93]. Use the neural network and fuzzy approach to detect the white matter, gray matter, celegreel spinal fluid and tumor for segmentation of magnetic resonance inference (MRI) images was proposed by [94].

\subsection{Hybrid Domain}

In the previous paragraphs, various methods were proposed for the image compression using fractal theory in spatial domain, frequency domain and soft-computing domain. Similarly, various researchers combined the relevant domains for better compression of images. By using spatial domain and frequency domain, the DCT approach along with fractal theory was proposed by [95] [96][97]. Applied the wavelet technique to compress the radiology images were given by [98]. The DCT and interpolation approach for FIC was proposed by [99][100] and used the wavelet approach for FIC by [101][102]. Similarly, edge extraction approach using fractal and wavelet was proposed by [103].

With the combination of spatial domain and softcomputing domain, defined the block features in spatial domain and used the fuzzy logic for classification of domain blocks by [104][105]. Used the image feature for better matching and neural network to reduce the mapping error for best match by [106][107]. Similarly, the spatial correlation and genetic algorithm for FIC was given by [108].

With the incorporation of frequency domain and softcomputing domain, the genetic algorithm used to separate the population into superior clan and inferior clan and then used the DCT approach for compression by [109] and for FIC, [110] used the fuzzy c-mean to separate the domain blocks into clusters and DCT approach to map the rangedomain.

\section{RESULTS AND DISCUSSIONS}

From the above literature review, the two major findings are: fractal dimension approach to measure the image complexity and fuzzy logic for image processing. Both the findings are sufficiently suitable for future expansion to compress the medical images using fractal theory. The two approaches are discussed in the following sub-sections:

\subsection{Fractal Dimension}

FD is an approach to identify the complexity of an image. In this context, the FD technique for texture segmentation by [111][112] and developed a fabric inspection system using fractal image analysis by [113]. The FD approach to identify the image features such as texture, roughness, smoothness and solidity by [114]. Similarly, the FD to calculate the feature vector of an image to classify them by [115] and classified the traffic images using FD by [116]. For FD calculation, various researchers have been proposed different methods. In this way, estimated the FD using box-counting was proposed by [117] and improved the box-counting approach by [118]. Use the local fractal features to calculate the fractal intrinsic dimension by [119]. A new algorithm for calculating the FD using triangular prism method was given by [120] and used the roughness of image to estimate the FD by [121]. In image compression, FD segmentation based FIC were proposed by [17][122]. Similarly, the FIC using local FD were given by [123][124] and [22] was proposed the vector quantization based image coding, where FD was used to partition the image and achieved high compression ratio.

From the above discussed FD approaches, some contributor results are shown. The results shown in Figure 2 , given by [120], clearly described that the geometric-step (varying coverage) method resulted in higher fluctuations in the average local fractal dimensions across windows. The results of Table 1, given by [118], shows the FD values of all the three methods along with proposed method for the three images of the sizes $512 \times 512$, $256 \times 256$ and $128 \times 128$ pixels. The results of table 2 , given by [121], shows that the FD, obtained by the roughness method, is little vary in comparison with the corresponding hausdorff dimension. The tabulated values of Table 3, given by [114], clearly substantiate the fact that, the fractal dimension of any given image will progressively increase with its roughness.

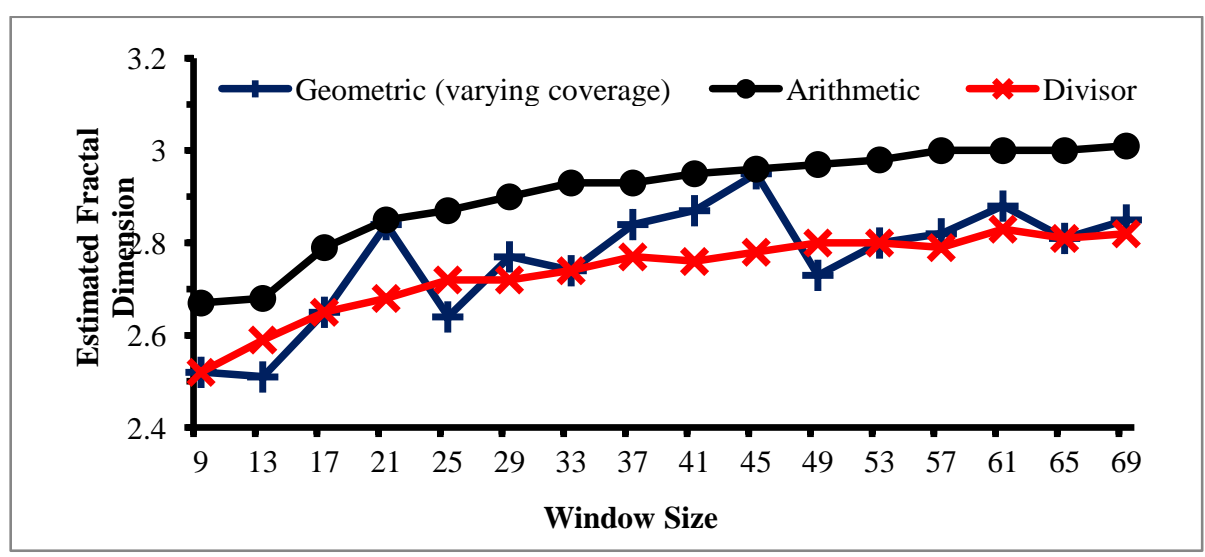

Fig 2: Estimated Fractal dimension with varying window size by [120] 
Table 1. Computational fractal dimensions of the remote images in different sizes by [118].

\begin{tabular}{|c|c|c|c|c|c|}
\hline \multirow{2}{*}{ Image } & $\begin{array}{c}\text { Image } \\
\text { size } \\
\text { (pixel) }\end{array}$ & DBC & $\begin{array}{c}\text { Improved } \\
\text { DBC }\end{array}$ & Pent & Kell \\
\hline \multirow{4}{*}{ RS1 } & $512 \times 512$ & 2.51 & 2.62 & 2.25 & 2.43 \\
\cline { 2 - 6 } & $256 \times 256$ & 2.55 & 2.64 & 2.83 & 2.54 \\
\cline { 2 - 6 } & $128 \times 128$ & 2.54 & 2.65 & 3.11 & 2.57 \\
\hline \multirow{4}{*}{ RS2 } & $512 \times 512$ & 2.34 & 2.42 & 2.32 & 2.23 \\
\cline { 2 - 6 } & $256 \times 256$ & 2.36 & 2.43 & 2.98 & 2.28 \\
\cline { 2 - 6 } & $128 \times 128$ & 2.38 & 2.44 & 3.38 & 2.31 \\
\hline \multirow{3}{*}{ RS3 } & $512 \times 512$ & 2.34 & 2.47 & 2.34 & 2.25 \\
\cline { 2 - 6 } & $256 \times 256$ & 2.35 & 2.49 & 2.82 & 2.38 \\
\cline { 2 - 6 } & $128 \times 128$ & 2.31 & 2.48 & 3.14 & 2.32 \\
\hline
\end{tabular}

Table 2. Compare fractal dimension with Hausdorff dimension [121]

\begin{tabular}{|c|c|c|c|}
\hline Fractal & A & $\begin{array}{c}\text { Roughness } \\
\text { dimension }\end{array}$ & $\begin{array}{c}\text { Hausdorff } \\
\text { dimension }\end{array}$ \\
\hline $\begin{array}{c}\text { Random dust } \\
\text { (non-fractal) } \\
D=2\end{array}$ & $1.990(2)$ & $0.010(2)$ & 0 \\
\hline $\begin{array}{c}\text { Sierpinski } \\
\text { carpet }\end{array}$ & $0.097(4)$ & $1.903(4)$ & 1.8928 \\
\hline $\begin{array}{c}\text { Sierpinski } \\
\text { triangle }\end{array}$ & $0.428(3)$ & $1.572(3)$ & 1.5850 \\
\hline $\begin{array}{c}\text { Standard } \\
\text { cantor set }\end{array}$ & $0.382(3)$ & $0.618(3)$ & 0.6309 \\
\hline $\begin{array}{c}\text { Menger } \\
\text { sponge }\end{array}$ & $0.288(1)$ & $2.712(1)$ & 2.7268 \\
\hline $\begin{array}{c}\text { Sierpinski } \\
\text { tetrahedron }\end{array}$ & $1.092(24)$ & $1.908(24)$ & 2 \\
\hline $\begin{array}{c}\text { Random dust } \\
\text { (non-fractal) } \\
D=4\end{array}$ & $3.886(50)$ & $0.114(50)$ & 0 \\
\hline
\end{tabular}

Table 3. Fractal Dimensino of currupted images lena, bird and rice with various level [114]

\begin{tabular}{|c|c|c|c|}
\hline \multirow{2}{*}{$\begin{array}{c}\text { Noise } \\
\text { Density }\end{array}$} & \multicolumn{3}{|c|}{ Fractal Dimension } \\
\cline { 2 - 4 } & lena & Bird & rice \\
\hline $10 \%$ & 2.8691 & 2.8716 & 2.8466 \\
\hline $20 \%$ & 3.8701 & 2.8721 & 2.8474 \\
\hline $30 \%$ & 2.8707 & 2.8723 & 2.8476 \\
\hline $40 \%$ & 2.8711 & 2.8728 & 2.8487 \\
\hline $50 \%$ & 2.8715 & 2.8731 & 2.8494 \\
\hline $60 \%$ & 2.8717 & 2.8729 & 2.8496 \\
\hline $70 \%$ & 2.8721 & 2.8733 & 2.8506 \\
\hline $80 \%$ & 2.8734 & 2.8739 & 2.8509 \\
\hline $90 \%$ & 2.8739 & 2.8741 & 2.8520 \\
\hline
\end{tabular}

\subsection{Fuzzy Logic in Image Processing}

The use of FL in image processing described the natural texture of image using fuzzy based FD and classified them by [75] and implemented the local fuzzy fractal dimension for medical images by [125]. Similarly, applied the fuzzy logic on domain blocks for classification by [126][104] and applied the fuzzy logic along with DCT, to speed up the FIC by [110]. The fuzzy based clustering technique for similar images by [76] and the fuzzy technique to detected the edges of various images by [77][78]. The application of fuzzy logic techniques for image binarization, edge detection and geometric measurement were described by [79] and the fuzzy based contrast enhancement technique for images by [80]. Along with all the methods, a new fuzzy c-means based clustering method was successfully applied for FIC by [105].

From the above discussed fuzzy based methods, some contributor results are given here. The crisp approach tends to discard more information, whereas the fuzzy approach tends to preserve more image information by [78] is depicted in Figure 3, in which the proposed model does not include uncertainty generated by human perception of complexity. Therefore, the image complexity measure provided by the procedure is very consistent and can applicable for image edge detection task. The comparative experiment results by [104] are shown in Table 4 . The results by [105] are depicted in Table 5 and Figure 4-5, shows that the proposed method for FIC is faster and remains better decoding image quality and can be applied to any image with different texture complexity, especially does better for an image with more complex texture.

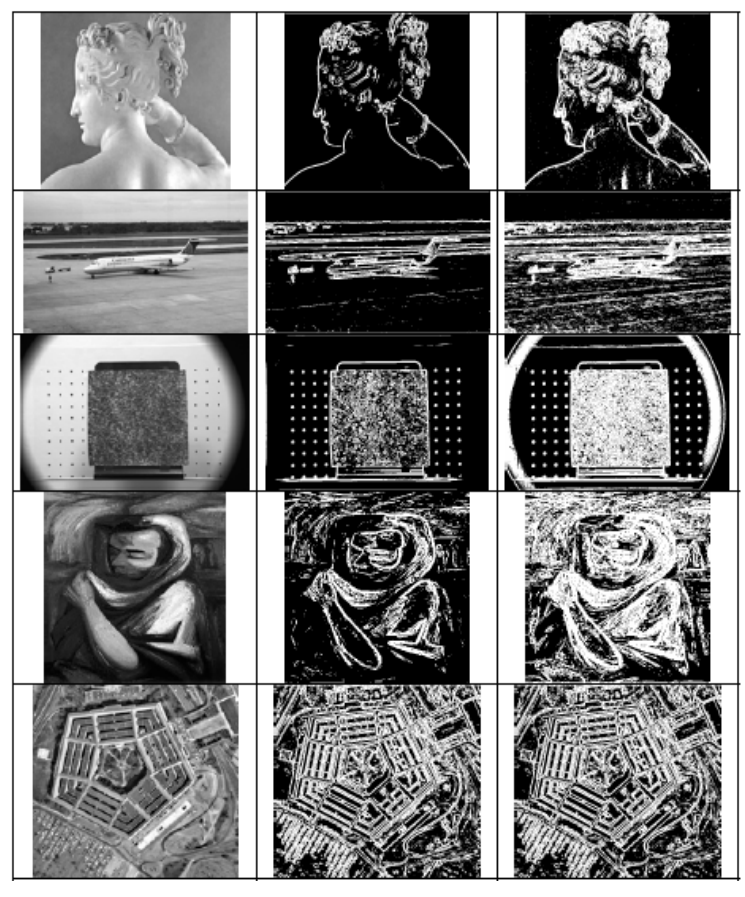

(a)

(b)

(c)

Fig 3: Edge detection based on image complexity a) Original b) Crisp complexity) Fuzzy based complexity by $[78]$ 


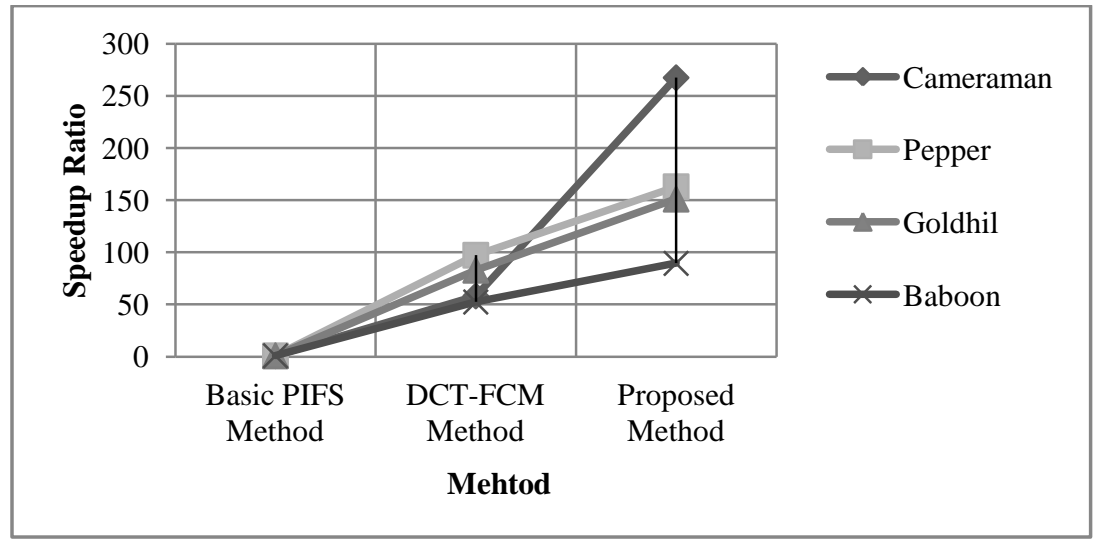

Fig 4: Comparison between the proposed method and other methods using speed-up ratio [105]

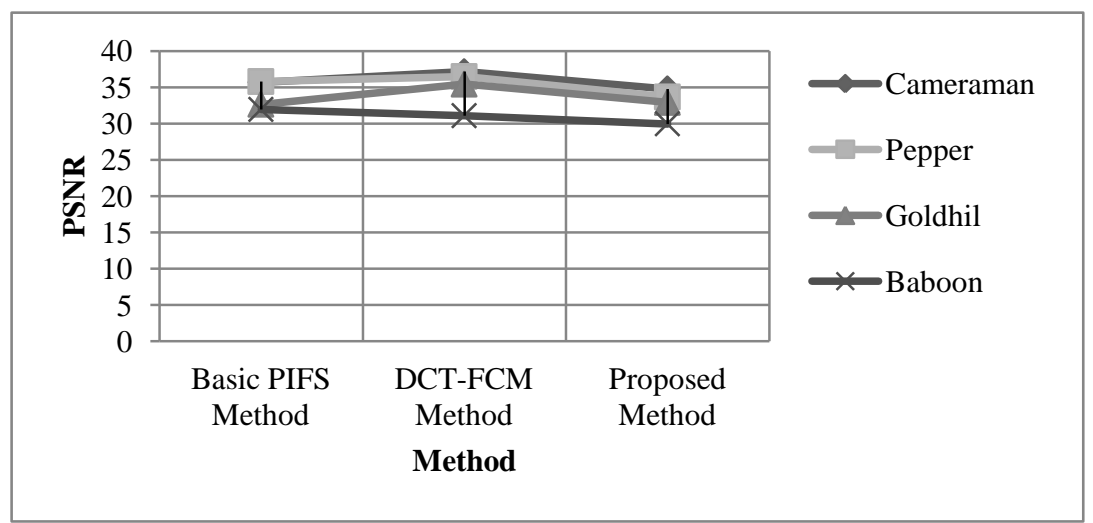

Fig 5: Comparison between the proposed method and other methods using PSNR factor [105]

Table 4. Experiments results of different encoding methods for Splash image [104]

\begin{tabular}{|l|c|c|c|}
\hline $\begin{array}{c}\text { Encoding } \\
\text { method }\end{array}$ & $\begin{array}{c}\text { Compression } \\
\text { ratio }\end{array}$ & $\begin{array}{c}\text { PSNR } \\
\text { (dB) }\end{array}$ & $\begin{array}{c}\text { Encoding } \\
\text { time (s) }\end{array}$ \\
\hline $\begin{array}{l}\text { Standard } \\
\text { method }\end{array}$ & 22.45 & 35.12 & 150 \\
\hline $\begin{array}{l}\text { Proposed } \\
\text { Method }\end{array}$ & 21.26 & 35.01 & 10 \\
\hline $\begin{array}{l}\text { Jacquin } \\
\text { method }\end{array}$ & 21.00 & 34.25 & 76 \\
\hline $\begin{array}{l}\text { Fisher } \\
\text { method }\end{array}$ & 15.51 & 33.17 & 12 \\
\hline
\end{tabular}

Table 5. Comparison the basic PIFS method with proposed FCM method for the image size $512 \times 512$ of $4 \times 4$ fixed sized block [105]

\begin{tabular}{|c|c|c|c|c|c|c|}
\hline \multirow{2}{*}{$\begin{array}{c}\text { Image } \\
\text { Name }\end{array}$} & \multicolumn{2}{|c|}{$\begin{array}{c}\text { Basic PIFS } \\
\text { Method }\end{array}$} & \multicolumn{2}{c|}{$\begin{array}{c}\text { DCT-FCM } \\
\text { Method }\end{array}$} & \multicolumn{2}{c|}{$\begin{array}{c}\text { Proposed } \\
\text { Method }\end{array}$} \\
\cline { 2 - 7 } & $\begin{array}{c}\text { Time } \\
\text { (s) }\end{array}$ & $\begin{array}{c}\text { PSNR } \\
\text { (dB) }\end{array}$ & $\begin{array}{c}\text { Coding } \\
\text { Time } \\
\text { (s) }\end{array}$ & $\begin{array}{c}\text { PSNR } \\
\text { (dB) }\end{array}$ & $\begin{array}{c}\text { Coding } \\
\text { Time } \\
\text { (s) }\end{array}$ & $\begin{array}{c}\text { PSNR } \\
\text { (dB) }\end{array}$ \\
\hline $\begin{array}{c}\text { Camera } \\
\text {-man }\end{array}$ & 3636 & 35.67 & 62.39 & 37.14 & 13.59 & 34.77 \\
\hline Pepper & 3644 & 35.78 & 37.41 & 36.47 & 22.35 & 33.66 \\
\hline Goldhill & 3624 & 32.65 & 43.66 & 35.42 & 23.98 & 32.90 \\
\hline Baboon & 3662 & 31.94 & 69.66 & 31.08 & 40.87 & 29.96 \\
\hline
\end{tabular}

From the above discussions, the computation method of FD could be affected by some parameters as the computation algorithm used with its parameter variation As a result, it is difficult to know whether the observed difference in computed FD values is a result of true difference in image texture or a result of certain arbitrary decision made during the estimation process. Therefore the choice of a method is an important issue in the computation of FD, which suggested that some results could be improved. Similarly, the fuzzy logic could be used to calculate FD and use it for domain blocks classification to speed up the encoding process of FIC. And also the fuzzy logic could be applied for texture segmentation, edge detection, and image enhancement.

The above two fruitful techniques, fractal dimension and fuzzy logic, are consider as to provides an innovative idea for fractal theory based medical image compression method to decrease the encoding time, increase the compression ratio and maintain the image fidelity.

\section{CONCLUSIONS}

The essence of FIC consists of catching the piecewise selfsimilarity existing in an image. For constructing a fractal representation of an image, it is expected to provide an efficient representation for natural images. Most authors assumed that natural images exhibit significant "selfsimilarity". For the FIC, the main key problem with any model would be to provide a good control mechanism for solving the local inverse problem to selecting the best transformation between two blocks without having to perform a complete search through a large pool of transformations. If the selection of the best domain block may be hard to predict, it is desirable to find the best matching transformation with little computation effort. 
Fractal geometry provides a powerful technique for the characterization of many images and has been applied to image compression. The applicability of the fractal theory in image analysis comes from the fact that the imaged objects are discontinuous, complex and fragmented. The significance and the advantages of this theory compared in three domain: spatial, frequency and soft-computing.

In this paper, various FIC techniques such as predictive coding, classified transform coding, fuzzy based coding, neural network based coding, and vector quantization with different partitioning methods such quad-tree and horizontal-vertical have been discussed.

Fractal analysis is often brought to the evaluation of the FD which allows having a global description of the homogeneities in the image. Its efficiency has been demonstrated in classification experiments where it was used as an additional texture parameter for accelerate the fractal image encoding.

Fuzzy logic efficiency has been demonstrated in texture analysis of images, image segmentation, edge detection, image enhancement. In FIC, it has been used as a classification of domain blocks.

As a conclusion of this study, the integration of FD and fuzzy logic could be an efficient technique to deal with medical image compression and also to enhance the reconstructed quality of compressed medical images with high compression ratio.

\section{REFERENCE}

[1] Kreyszig E. Introductory Functional Analysis with Applications. John Wiley \& Sons, New York, 1978.

[2] Pratt W K. Vector space formulation of twodimensional signal processing operations. Computer Graphics and Image Processing, 1975, 4(3):1-24.

[3] Fisher Y. Fractal Image Compression: Theory and Applications. Springer-Verlag, New York, USA, 1995.

[4] Barnsley M. Fractal Everywhere, Academic Press. San Diego, USA, 1992.

[5] Peitgen H O, Jurgens H, and Saupe D. Chaos and Fractals: New Frontiers of Science. Springer-Verlag, New York, USA, 1992

[6] Lu N. Fractal Imaging. Academic Press. San Diego, USA, 1997.

[7] Jacquin. A. E. 1992. Image Coding Based on a Fractal Theory of Iterated Contractive Image Transform. Journal of IEEE Transaction on Image Processing, 1(1): 18-30.

[8] Hürtgen. B., Müller. F., and Stiller. C. 1993. Adaptive Fractal Coding of Still Pictures. International Symposium, Switzerland :1-4.

[9] Jacquin. A. E. 1993. Fractal Image Coding: A Review. Proceeding of IEEE, 81(10): 1451-1465.

[10] Jun. Y., Shuzhen. C., Qiang. S., and Xiaoan. S. 1997. Fractal Block Coding: A New Method. Journal of Natural Science, 2(1): 63-67

[11] Kung. C. M., Yang. W. S., Ku. C. C., and Wang. C. Y. 2008.Fast Fractal Image Compression Base on
Block Property. IEEE International Conference on Advanced Computer Theory and Engineering: 477481

[12] Qin. F, Min. J, Guo. H, and Yin. D. 2009. A Fractal Image Compression Method Based on Block Classification and Quadtree Partition. IEEE Computer Society World Congress on Computer Science and Information Engineering: 716-719.

[13] Saupe.D. and Jacob. S. 1997.Variance-Based Quadtrees in Fractal Image Compression. Journal Electronic Letter, 33(1): 46-48.

[14] Huang. W. Y. and Wen. M. 2003. Fractal Image Compression with Variance and Mean. Proceedings of International Conference on Multimedia and Expo,1: 353-356.

[15] Zhou. W., Huaqiu. D., and Yinglin. Y. 1997. Fractal Block Coding In Residue Domain. Journal of Electronics, 14(3): 236-240.

[16] Truong. T. K., Kung. C. M., Jeng. J. H., and Hsieh. M. L. 2004. Fast fractal image compression using spatial correlation. Elsevier Journal of Chaos, Solutions and Fractals, 22: 1071-1076.

[17] Jang.J. andRajala. S. A. 1990. Segmentation Based Image Coding Using Fractals and the Human Visual System. International Conference on Acoustics, Speech, and Signal Processing, 4: 1957-1960.

[18] Hartenstein. H., Ruhl. M., and Saupe. D. 2000. Region-Based Fractal Image Compression Journal. IEEE Transaction of Image Processing, 9(7): 11711184.

[19] Dugelay.J. andGersho. A. 1997. Enhanced Fractal Image Coding by Combining IFS and VQ. Conference Proceeding of International Conference on Image Processing, 3: 726-729.

[20] Hamzaoui. R. and Saupe. D. 2000. Combining Fractal Image Compression and Vector Quantization. IEEE Transaction of Image Processing, 9(2): 101-137.

[21] Chen. S. S., Yang. C. B., and Huang. K. 2002. Fractal Image Compression based on Intra-block Variance Distribution and Vector Quantization. Journal of Optical Engg.,41(11): 2824-2830.

[22] Moyamoto.T., Suzuki.Y.,Saga.S., and Maeda. J. 2005 Vector Quantization of Images Using Fractal Dimensions. IEEE Mid-Summer Workshop on Soft Computing in Industrial Applications

[23] Chaurasia.V. andSomkuwar. A. 2010. Improved Suitable Domain Search for Fractal Image Encoding. International Journal of Electronic Engineering Research, 2(1): 1-8.

[24] Davoine.F., Antonini.M., Chassery.J.M., and Barlaud. M. 1996. Fractal Image Compression Based on Delaunay Triangulation and Vector Quantization. Journal IEEE Transaction on Image Processing, 5(2): 338-345.

[25] Yisong. C., Jian. L., Zhengxing. S., and Fuyan. Z. 2002. Greylevel Difference Classification Algorithm in Fractal Image Compression. Journal of Computer Science \& Technology, 17(2): 236-239 
[26] Prasad. V. R., Vaddella, Babu. R., and Inampudi. 2007. Adaptive Gray Level Difference to Speed up Fractal Image Compression. IEEE International Conference on Signal Processing, Communications and Networking: 253-258

[27] Saupe. D. 1994. Breaking the Time Complexity of Fractal Image Compression. International Conference Germany: 1-8.

[28] Saupe. D. 1995. Accelerating Fractal Image Compression by Multi-Dimensional Nearest Neighbor Search. Conference Proceedings on Data Compression Conference: 222-231.

[29] Xu.C. and Zhang. Z. 2001. A Fast Fractal Image Compression Coding Method. Journal of Shanghai University (English Edition), 5(1): 57-59.

[30] Ghosh. S. K., Mukherjee. J., and Das. P. P. 2004. Fractal image compression: a randomized approach Elsevier Journal Pattern Recognition Letters, 25: 1013-1024.

[31] Cardinal. J. 2001. Fast Fractal Compression of Greyscale Images. IEEE transaction of Image Processing, 10(1): 159-164.

[32] Sze.C. and Wong. M. 2002. Adaptive Approximate Nearest Neighbor Search for Fractal Image Compression. IEEE Transaction of Image Processing, 11(6): 605-615

[33] Xing. C., Ren. Y., and Li. X. 2008. A Hierarchical Classification Matching Scheme for Fractal Image Compression. IEEE Congress on Image and Signal Processing, 1: 283-286.

[34] Thomas. L. and Deravi. F. 1995. Region-Based Fractal Image Compression Using Heuristic Search. IEEE Transaction on Image Processing, 4(6): $832-838$

[35] Saupe. D., Ruhl. M., Hamzaoui. R., Grandi. L., and Marini. D. 1998. Optimal Hierarchical Partitions for Fractal Image Compression. Proceedings of International Conference on Image Processing, 1: 737-741.

[36] Chao. H. C. and Chieu. B. C. 1998. Fractal Image Coding Using Projection-Based Classification and Variable Shape Matching. Journal of the Chinese Institute of Engineers, 21(5): 507-520.

[37] Chu. H. T. and Chen. C. C. 2001. Accelerating Fractal Image Compression with a Real-Time Decoder. Journal of Information Science and Engg., 17:417-427.

[38] Wang. X. Y. and Wang. S. G. 2008. An improved nosearch fractal image coding method based on a modified gray-level transform. Science Direct Journal of Computer \& Graphics, 32: 445-450.

[39] Lai.C. M., Lam. K. M., and Siu. W. C. 2003. A Fast Fractal Image Coding Based on Kick-Out and Zero Contrast Conditions. IEEE Transaction of Image Processing, 12(11): 1398-1403.

[40] He. C., Yang. S. X., and Xu. X. 2004. Fractal Image Compression Based on One-Norm of Normalized Block. Electronic Letter Journal, 40(17): 1-2.
[41] Chen. H. N., Chung. K. L., and Hung. J. E. 2009. Novel fractal image encoding algorithm using normalized one-norm and kick-out condition. Elsevier Journal of Image and Vision Computing, 28: 518-525.

[42] Saupe. D. and Ruhl. M. 1996. Evolutionary Fractal Image Compression Conference. Proceedings of International Conference on Image Processing, 1: 129-131.

[43] Zhou. W. and Yinglin. Y. 1997. Dynamic Fractal Transform with Applications to Image Data Compression. Journal of Computer Science and Technology, 12(3): 202-209.

[44] Yixia. L., Quanfan. Z., and Jun. L. 1998. Static Image Compression Based on Fractal Theory. Journal of Shanghai University, 2(3): 252-255.

[45] Muruganandhama. A. and Wahidabanub. R. S. D. 2010.Adaptive Fractal Image Compression using PSO. Elsevier Journal Procedia Computer Science,2 338-344.

[46] Chakrapani.Y. andSoundararajan. K. 2010 Implementation of fractal image compression employing particle swarm optimization. World Journal of Modeling and Simulation, 6(1): 40-46.

[47] Jeng. J. H., Tseng. C. C., and Hsieh. J. G.2009. Study on Huber Fractal Image Compression. IEEE Transaction on Image Processing, 18(5): 995-1003.

[48] Addison. P. S. 2005. Fractals and Chaos.IOP Publication.

[49] Ong. G. H., Chew. C. M., and Cao.Y. 2001. A Simple Partitioning Approach to Fractal Image Compression. ACM Conference SAC 2001: 301-305.

[50] Žumbakis.T. and Valantinas. J. 2006. A Modified Approach to Fractal Encoding of Binary Images. Journal Information Technology and Control, 35(1):13-18

[51] Sze. C. and Pi. M. 2001. Fast Fractal Image Encoding Based on Adaptive Search. Journal IEEE Transaction of Image Processing, 10(9): 1269-1277.

[52] Tonga. C. S. and Pi. M. 2003. Analysis of a hybrid fractal-predictive-coding compression scheme.Journal (Elsevier) Signal Processing: Image Communication, 18: 483-495

[53] Stapleton. W. A., Mahmoud. W., and Jackson. D. J. 1996. A Parallel Implementation of a Fractal Image Compression Algorithm. Conference Proceedings of the Twenty-Eighth Southeastern Symposium on System Theory: 332-336.

[54] Saupe. D. 1996. The Futility of Square Isometric in Fractal Image Compression Conference. Proceedings of International Conference on Image Processing, 1: 161-164

[55] Kumar.S. and Jain. R. C. $1997 . \quad$ Low Complexity Fractal-Based Image Compression Technique. IEEE Transaction of Consumer Electronics, 43(4): 987-992.

[56] Tseng. C. C., Hsieh. J. G., and Jeng. J. H. 2008. Fractal image compression using visual-based particle 
swarm optimization. Elsevier Journal of Image and Vision Computing, 26: 1154-1162.

[57] Liang. J. Y., Chen. C. S., Huang. C. H. and Liu. L. 2008. Lossless Compression of Medical Images using Hilbert space-filling curves. Elsevier Journal of Computerized Medical Imaging and Graphics, 32: 174-182.

[58] Wohlberg. B. E. and Jager. G. D. $1995 . \quad$ Fast Image Domain Fractal Compression by DCT Domain Block Matching. Electronic Letter, 31 (11): 869-870.

[59] Belloulata. K., Stasinski. R., and Konrad. J. 1999. Region Based Image Compression Using Fractal and Shape Adaptive DCT. Proceeding of International Conference on Image Processing, 2: 815-819.

[60] Truong. T. K., Jeng. J. H., Reed. I. S., Lee. P. C., and Li. A. Q. 2000. A Fast Encoding Algorithm for Fractal Image Compression Using the DCT Inner Product. IEEE Transaction of Image Processing, 9(4): 529-535.

[61] Wang. C. C., and Kao. J. Y. 2004. A Fast Encoding Algorithm for Fractal Image Compression. IEICE Electronic Express, 1(12): 352-357.

[62] Wang. C. C., Lin. L. C., and Tsai. S. H. 2004. Fast Fractal Encoding Algorithm Using the Law of Cosine. The2004 47th Midwest Symposium on Circuits and Systems, 1: 233-236.

[63] Duha. D. J., Jengb. J. H., and Chena. S.Y. 2005. DCT based simple classification scheme for fractal image compression. Elsevier Journal of Image and Vision Computing, 23: 1115-1121.

[64] Žumbakis. T., and Valantinas. J. 2005. The Use of Image Smoothness Estimates in Speeding up Fractal Image Compression. Proceeding of 14th Scandinavian Conference (SCIA 2005), 3540: $1167-$ 1176.

[65] Ponomarenko. N. N., Egiazarian. K., Lukin. V. V, and Astola. J. T. 2007. High-Quality DCT-Based Image Compression Using Partition Schemes. IEEE Journal of Signal Processing Letter, 14(2):105-108.

[66] Saupe D. and Hartenstein H. 1996.Lossless acceleration of fractal image compression by fast convolution. International Conference on Image Processing, 1: 185-188.

[67] de Oliveira. J. F. L., Mendonca. G. V., and Dias. R. J. 1998. A Modified Fractal Transformation to Improve the Quality of Fractal Coded Images. IEEE Proceeding of International Conference on Image processing, 1: 756-759.

[68] Kim. I. K. and Park. R. H. 1998. A Fast Encoding Method Without Search for Fractal Image Compression. Proceedings of the 1998 IEEE International Conference on Acoustics, Speech and Signal Processing, 5: 2625-2628.

[69] Davis. G. M. 1998. A Wavelet Based Analysis of Fractal Image Compression. IEEE Transaction on Image Processing, 7(2): 100-112.

[70] Endo. D., Hiyane. T., Atsduta. K., and Kondo. S. 1998. Fractal Image Compression by the
Classification in the Wavelet Transform Domain. Proceedings of the 1998 IEEE International Conference on Image Processing, 1: 788-792.

[71] Deyuan. C., Enjie. L., and Guofang. T. 2008. A New Fractal Coder for SAR Imagery with High Frequency Energy Matching. 11th IEEE International Conference on Communication Technology Proceeding: 680-683.

[72] Yadav. D. M. and Bormane. D. S. 2008. Evaluation of Pure Fractal and Wavelet-Fractal Image Compression Techniques . International Journal on Graphics, Vision and Image Processing, 8(2): 37-44.

[73] Hien. V. T. 2009. A New Wavelet-Fractal Image Compression Method. Proceeding of 13th International Conference on Knowledge-Based and Intelligent Information and Engineering Systems, 5711: 161-168.

[74] Jeng. J. H. and Shyu. J. R. 2000. Fractal image compression with simple classification scheme in frequency domain, Journal of Electronic Letter, 36(8): $1-2$

[75] Bas. O. Y. and Erkmen. A. M. 1995. A new approach to the fractal based description of natural textures: the fuzzy fractal dimension. IEEE International Conference on Systems, Man and Cybernetics, 4: 3232-3237.

[76] Keller. A. and Klawonn. F. 1999. Context Sensitive Fuzzy Clustering.18th International Conference of the North American Fuzzy Information Processing Society: $347-351$

[77] Ignacio.M., Chacon.M., L. E. Aguilar. 2001. A Fuzzy Approach to Edge Level Detection. IEEE International Fuzzy System Conference, 809-812

[78] Ignacio. M., Delia. A., Rodriguez. R. S. 2007. A Fuzzy Approach on Image Complexity Measure. Journal of Computation System, 10(3): 268-284.

[79] Krishnan. M. H. and Viswanathan. R. 2010 Application of Advanced Fuzzy Logic Techniques in Fuzzy Image Processing Scheme . Journal of Advance in Fuzzy Mathematics, 5(1): 71-76.

[80] Balasubramaniam J., Kakarla. V. V. D. L., Narayana, and Vetrivel. V. 2011. Fuzzy Inference System based Contrast Enhancement, Atlantis Press: 311-318.

[81] Pal. S. and King. R. A.1981. Image Enhancement using Smoothing with Fuzzy Sets. Journal IEEE Transaction On System, Man and Cybernetics, 11(7): 494-501.

[82] Loe. K. F., Gu. W. G., and Phua. K. H. 1997. SpeedUp Fractal Image Compression with a Fuzzy Classifier. Elsevier Journal of Signal Processing: Image Communication.10: 303-311.

[83] Welstead. S. 1997. Self-Organizing Neural Network Domain Classification for Fractal Image Coding. International Conference on Artificial Intelligence and Soft Computing: 248-251.

[84] Lee. S. J., Wu. P.Y., and Sun. K. T. 1998. Fractal Image Compression Using Neural Network 
Proceedings of IEEE International Joint Conference on Neural Networks, 1: 613-618.

[85] Sun. K. T., Lee. S. J., and Wu. P. Y.2001. Neural Network Approaches to Fractal Image Compression and Decompression. Elsevier Journal of Neurocomputing, 41: 91-107.

[86] Lototskiy. R. V. 2003.Images Fractal Compression Optimization by Means of Artificial Kohonen Neural Networks. Journal of Automation and Information Sciences, 35(1): 50-61.

[87] Chakrapani.Y. andRajan. K. S. 2008. Implementation of fractal image compression employing artificial neural networks. World Journal of Modeling and Simulation, 4(4): 287-295.

[88] Vences. L. A. and Rudomin. I. 1997. Genetic Algorithms for Fractal Image and Image equence Compression. Proceeding of Compaction Visual: 110.

[89] Mitra. S. K., Murthy. C. A., and Kundu. M. K. 1998. Technique for Fractal Image Compression Using Genetic Algorithm. IEEE Transaction on Image Processing, 7(4): 586-593.

[90] Wu. M. S., Jeng. J. H., and Hsieh. J. G. 2007. Schema genetic algorithm for fractal image compression. Science Direct Journal of Engineering Applications of Artificial Intelligence, 20:531-538.

[91] Xi. L. and Zhang. L. 2007. A Study of Fractal Image Compression Based on an Improved Genetic Algorithm. International Journal of Nonlinear Science, 3(2): 116-124.

[92] Lalitha. E. M. and Satish. L. 1998. Fractal Image Compression for Classification of PD Sources. IEEE Transaction on Dielectrics and Electrical Insulation, 5(4): 550-557.

[93] Chakrapani.Y. and Soundararajan. K. 2009. Adaptive Neuro-Fuzzy Inference System based Fractal Image Compression. International Journal of Recent Trends in Engg., 2(1): 161-168.

[94] SatishK.S., Moorthi.M., Madhu.M., and Amutha. R.2010. An improved method of segmentation using Fuzzy-Neuro Logic. Second International Conference on Computer Research and Development: 671-675.

[95] Zhao.Y. and Yuan. B. 1994. Image Compression using fractals and discrete cosine transform. Electronic Letter, 30(6): 474-475.

[96] Melnikov.G. and Katsaggelos. A. K. 2002. A Jointly Optimal Fractal/DCT Compression Scheme. IEEE Transaction on Multimedia, 4(4): 413-422.

[97] Zhou. Y. M., Zhang. C., and Zhang. Z. K. 2009. An efficient fractal image coding algorithm using unified feature and DCT. Chaos, Solitons and Fractals: the interdisciplinary journal of Nonlinear Science, and Non-equilibrium and Complex Phenomena, 39(4): 1823-1830.

[98] Goldberg. M. A. 1997. Image Data Compression. Journal of Digital Imaging, 10(3): 9-11.
[99] Shi. Y., Gu. W., Zhang.L., and Chen. S. 1997. Some New Methods to Fractal Image Compression. Journal of Communication in Nonlinear Science \& Numerical Simulation, 2(2): 80-85.

[100] Zhongke.Y., Shaoguo.Y., and Deren. G. 1998. A New Fractal Image Coding Method. Journal of Electronics, 15(2): 125-129.

[101] Hebert. D. J. and Soundararajan. E. 1998. Fast Fractal Image Compression With Triangular Multiresolution Block Matching. Proceedings of International Conference of Image Processing, 1: 747 750 .

[102] Lin. S. C., Rui.F., Qiang. L. F., and Xi. C. 2007. A Novel Fractal Wavelet ImageCompression Approach. Journal of China University of Mining \& Technology, 17(1): 121-125.

[103] Zhu. Q. 2010.Edges Extraction Method based on Fractal and Wavelet. Journal of Computer, 5(2): 282289.

[104] Han. J. 2008. Fast Fractal Image Compression Using Fuzzy Classification. IEEE Fifth International Conference on Fuzzy Systems and Knowledge Discovery: 272-276

[105] Biswas A. K., Karmakar S., Sharma S., and Kowar M K. 2013.Fractal image compression by pixels pattern using fuzzy c-means. Journal of Engg. Research, 1(3): 109-121

[106] Zhou. Y., Zhang.C., and Zhang. Z. 2006. Improved Variance-Based Fractal Image Compression Using Neural Networks. Conference (Springer), ISNN: 575580 .

[107] Zhou. Y. M., Zhang. C., and Zhang. Z. K. 2008. Fast hybrid fractal image compression using an image feature and neural network. Science Direct Journal of Chaos, Solitons and Fractals, 37: 623-631.

[108] Wang. X. Y., Li. F. P., and Wang. S. G. 2009. Fractal Image Compression Based on Spatial Correlation and Hybrid Genetic Algorithm. Elsevier Journal of Visual Communication and Image Representation, 20: 505-510.

[109] Wu. M. S. and Lin. Y. L. 2010. Genetic algorithm with a hybrid select mechanism for fractal image compression. Elsevier Journal of Digital Signal Processing, 20: 1150-1161.

[110] Jaferzadeh. K., Kaini. K., and Mozaffari. S. 2012. Accelerating of Fractal Image Compression using Fuzzy Clustering and Discrete-Cosine Transformbased Metric. IET Journal of Image Processing, 6 (7): 1024-1030.

[111] Chaudhuri. B. B. and Sarkar. N. 1995. Texture Segmentation using Fractal Dimension. IEEE Journal of Transactions on Pattern Analysis and Machine Intelligence, 17(1): 72-77.

[112] Hsu.T.andKuo-Jui.H. 2008. Multi-resolution Texture Segmentation Using Fractal Dimension. IEEE International Conference on Computer Science and Software Engg., 6: 201-204 
[113] Conci.A.andProenc. C. B. 1998. A fractal image analysis system for fabric inspection based on a boxcounting method. Elsevier Journal of Computer Networks and ISDN Systems, 30: 18871895.

[114] Shanmigavadivu. P. and Sivakumar. V. 2012 Fractal Dimension Based Texture Analysis of Digital images. Elsevier Journal of Procedia Engineering, 38: 2981-2986.

[115] Min. Y., Sheng. Y. W., Bin. S., and Hong-hua. D. 2003. An image retrieval system based on fractal dimension. Journal of Zhejiang University Science, 4(4): 421-425.

[116] Cao. W. L., Shi. Z. K., and Feng. J. H. 2006. Traffic Image Classification Method based on Fractal Dimension. Proceeding of $5^{\text {th }}$ IEEE International Conference on Cognitive Informatics: 903-907.

[117] Morita. T. 2005. Fractal Dimension Estimators for a Fractal Process. Journal of the Korean Physical Society, 46(3): 631-637.

[118] Li. J., Du. Q., and Sun. C. 2009. An Improved BoxCounting Method For Image Fractal Dimension Estimation. Elsevier Journal of Pattern Recognition, 42: 2460-2469.

[119] Cai. Q. and Zhang. C. 2006. Estimating Fractal Intrinsic Dimension from the Neighborhood. Springer Journal of Advances in Neural Network, 3971: 1312 1318.
[120] Wenxuelu.J.and Lam. N. 2009.An Improved Algorithm for Computing Local Fractal Dimension Using the Triangular Prism Method. Elsevier Journal of Computer \& Geosciences, 35: 1224-1233.

[121] Bãlachowski. A., Ruebenbauer. K. 2009. Roughness Method to Estimate Fractal Dimension. ACTA PhysicaPolonica, 115(3): 636-340.

[122] Conci.A.and Aquino. F. R. 1999. Fractal Image Coding by Multi-Scale Selection Based on Block Complexity. Journal of Geometry Graphics, 3(1): 5765.

[123] Conci.A. and Aquino. F. R. 2005. Fractal Coding Based on Image Local Fractal DimensionJournal Computational \& Applied Mathematics, 24(1): 83-89.

[124] Valarmathi. M. L. and Anbumani. K. 2006. Non Iterative Fast Fractal Codec Using Local Fractal Dimension. GVIP Journal, 6(3): 1-5.

[125] Zhuang X., and Meng. Q. 2004. Local fuzzy fractal dimension and its application in medical image processing, Elsevier Journal of Artificial Intelligence in Medicine, 32: 29-36.

[126] Khaled, M. M. and Mohamed, T. E. 2002. A fast Fractal Image Coding approach employing Fuzzy Aggregation of Domain Blocks. Proceedings of the 6th Conference of Digital Image ComputingTechniques and Applications: 319-324. 